\title{
Chloroma of the Bladder: A Case Report of Leukemia Progression Presenting as Hematuria
}

\author{
Laurie K. Pearson $^{a}$ Ruchi Hamal ${ }^{b}$ Sanhong Yu ${ }^{c}$ Kenneth B. Miller ${ }^{a}$ \\ Felix Mensah $^{a}$ \\ aDivision of Hematology/Oncology, Tufts Medical Center, Boston, MA, USA; ${ }^{b}$ Department of \\ Hematology and Oncology, Mt Auburn Hospital, Cambridge, MA, USA; 'Department of Lab \\ Medicine and Pathology, Tufts Medical Center, Boston, MA, USA
}

\section{Keywords}

Myeloid sarcoma · Acute myelogenous leukemia - Extramedullary leukemia - Rare bladder neoplasms · Case report

\begin{abstract}
Myeloid sarcoma (MS) is a rare extramedullary manifestation of acute myelogenous leukemia (AML). The mass is composed of primitive myeloid cells that can occur in a variety of organs, most commonly the skin, lymph nodes, GI tract, bone, breast, and CNS. Involvement of the genitourinary tract is rare. Consensus on treatment of MS has not been established, but management typically involves systemic therapy, such as chemotherapy or allogeneic hematopoietic stem cell transplant as well as palliative local therapies such as radiation or surgery. Outcomes of MS using novel AML therapies, such as BCL-2 inhibitors or IDH inhibitors, remain undescribed. We describe a rare case of a 70 -year-old man presenting with MS of the urinary bladder complicating known secondary AML (RUNX1 and IDH2 mutated). Prior to development of bladder MS, the patient had received decitabine, enasidenib, and venetoclax. Following diagnosis, he was treated with cytarabine and venetoclax. To our knowledge, this is the first case of bladder MS treated with a BCL-2 inhibitor.
\end{abstract}

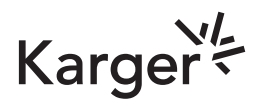




\section{Introduction/Background}

Acute myelogenous leukemia (AML) typically involves intramedullary proliferation of myeloid precursor cells. Extramedullary (EM) manifestations of AML are rare and include myeloid sarcoma (MS). MS is a solid EM mass composed of primitive myeloid cells that were initially described in 1811 by Burns [1]. In 1853, King [2] used the term chloroma to describe the greenish appearance that occurred as a result of myeloperoxidase in the blasts. As onethird of the masses appear gray or white, the term chloroma was replaced by granulocytic sarcoma or MS [3]. MS became a separate pathologic entity in the 2008 WHO classification of AML and myeloid neoplasms [4].

MS can present concurrently with AML, as relapse in a patient with previously treated AML, or precede the diagnosis of AML without evidence of leukemia in the blood or bone marrow [5]. In the latter case, without systemic treatment, overt leukemia develops after an average of 10.5 months [6]. Less frequently, MS may occur with the myelodysplastic syndromes or myeloproliferative neoplasms [7].

MS is seen in $<1 \%$ of all AML [8]. Risk factors associated with the development of MS in AML include the chromosomal abnormalities of $t(8 ; 21)$ and inversion (16); expression of cell surface markers CD56, CD2, CD4, and CD7; and the following AML subtypes: acute monoblastic/monocytic leukemia, acute myelomonocytic leukemia, and AML with maturation. Additional risk factors include poor nutritional status, decreased cellular immunity, high presenting leukocyte count, and decreased Auer rods [9]. MS is more common in men than in women (male-to-female ratio 1.42:1) and in younger patients (median age MS 55.8, median age all AML 66) [4].

Presenting signs and symptoms are due to the mass effect of the disease and involved organ dysfunction. The most frequently reported sites are the skin, lymph node, testis, GI tract, and bone [4].

Leukemic involvement of the urinary bladder is rare. Of 112 patients with EM relapse of AML after stem cell transplant, only 6 cases involved the bladder [10]. We report a case of MS of the urinary bladder presenting with gross hematuria and obstructive nephropathy in a patient with a known diagnosis of AML.

\section{Case Report}

A 70-year old man with a history of secondary AML (IDH2 and RUNX1 mutated) evolving from low-risk MDS with refractory anemia presented to our facility with new-onset hematuria. MDS was initially treated with erythropoietin-stimulating agents but progressed to AML after 20 months. Given the patient's poor performance status, he was deemed not a candidate for aggressive induction therapy and potentially curative allogeneic stem cell transplant, and treatment with the hypomethylating agent azacitidine was initiated with palliative intent. Following 4 cycles of azacitidine, he had disease progression with an increase in blasts on bone marrow aspiration and biopsy. Next-generation sequencing showed persistence of RUNX1 and IDH2 mutation. Cytogenetics showed normal male karyotype. Therapy was switched to decitabine and enasidenib with disease control for 9 months. He subsequently developed worsening cytopenia and increasing peripheral blasts, prompting a change in therapy to decitabine, venetoclax, and hydroxyurea with palliative intent. A bone marrow biopsy done to assess the response to venetoclax and decitabine showed marked hypercellularity with large immature appearing cells with moderate basophilic cytoplasm, delicate nuclear chromatin, and 1-4 round/oval nucleoli (shown in Fig. 1A). The blasts were positive for CD34 and c-kit immunohistochemical stains (shown in Fig. 1B, C).

\section{Karger'}



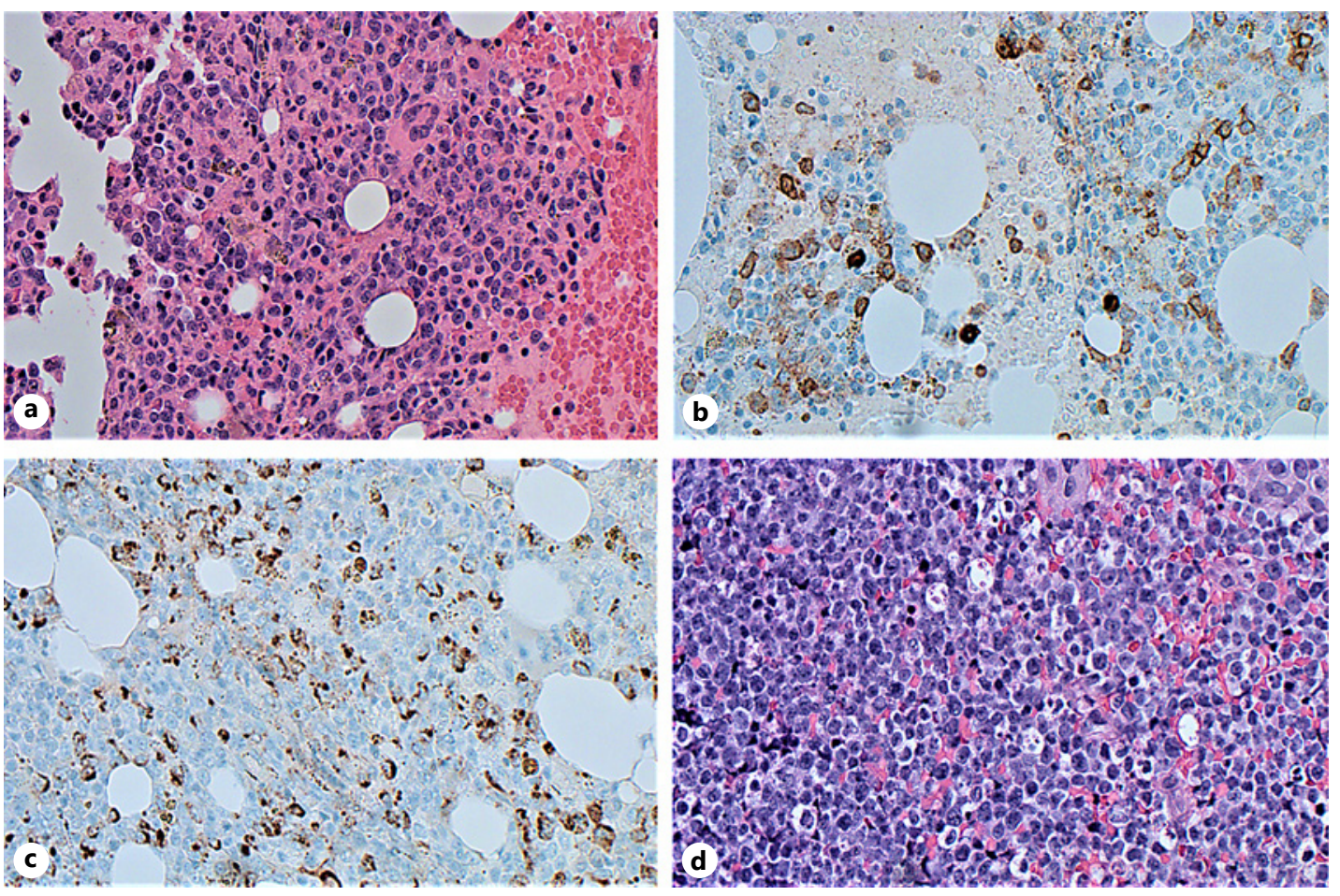

Fig. 1. a Bone marrow. Markedly hypercellular with large immature appearing cells with moderate basophilic cytoplasm, delicate nuclear chromatin, and 1-4 round/oval nucleoli (H\&E, ×400). b Bone marrow. CD34 immunohistochemical stain showing cytoplasmic positivity in tumor cells $(\times 400)$. c Bone marrow. CD-117 immunohistochemical stain on cell block section showing cytoplasmic positivity in tumor cells $(\times 400)$. d Bladder mass. Sheet of myeloblasts invading muscularis propria (H\&E stain, $\times 100)$.

After 10 months of disease control with decitabine and venetoclax, he presented to our facility reporting a 3-month history of progressive, intermittent, frank hematuria. Review of systems revealed urinary frequency, suprapubic pain, and generalized fatigue. CBC showed leukocytosis of $66 \mathrm{~K} / \mu \mathrm{L}$ (normal range 4.0-11.0 K/ $\mu \mathrm{L}$ ), anemia, with a hemoglobin level of $4.6 \mathrm{~g} / \mathrm{dL}$ (normal range 11.0-15.0 g/dL), and thrombocytopenia, with platelet count of $49 \mathrm{~K} / \mu \mathrm{L}$ (normal range $150-450 \mathrm{~K} / \mu \mathrm{L}$ ). The coagulation panel was normal. Urinalysis revealed $>100 \mathrm{RBCs}$ per hpf and $58 \mathrm{WBCs}$ per hpf. Creatinine was 2.73 $\mathrm{mg} / \mathrm{dL}$ (baseline: $0.90 \mathrm{mg} / \mathrm{dL}$, normal range $0.57-1.30 \mathrm{mg} / \mathrm{dL}$ ) concerning for obstructive uropathy. Blood and urine cultures were obtained, and empiric antibiotics were initiated. The patient was supported with red blood cell and platelet transfusions. A CT abdomen and pelvis without contrast showed an enlarged right kidney with stranding and associated moderate hydroureter/hydronephrosis, consistent with pyelonephritis, and asymmetric bladder wall thickening with adjacent fat stranding, suggestive of cystitis. Cystoscopy revealed a diffuse necrotic appearing bladder with areas of active bleeding and near obliteration of the ureteral orifices. Biopsies were taken, and a right nephrostomy tube was inserted.

The bladder mass obtained via transurethral bladder resection showed numerous loosely cohesive/noncohesive large cells with moderate basophilic cytoplasm, single round/oval nuclei, fine chromatin, and 1-4 distinct nucleoli invading the muscularis propria (shown in Fig. 1D). The bladder mass biopsy also showed numerous noncohesive myeloid cells invading the bladder wall against a background of benign von Brunner's nests. These myeloblasts showed fine nuclear chromatin, lobulated nuclei, and 2-4 nucleoli (data not shown). The tumor 
cells were positive for CD34 and negative for pankeratin immunohistochemical stains (data not shown). These findings were consistent with leukemic cells of myeloid lineage involving the bladder. Immunohistochemical stains revealed the blasts were strongly positive for CD34 and CD4. Next-generation sequencing revealed FLT3-ITD, IDH2, and RUNX1 mutations. Overall, findings were consistent with MS of the urinary bladder.

Treatment was changed to venetoclax and cytarabine $100 \mathrm{mg} / \mathrm{m}^{2}$ by continuous infusion. Blast count declined rapidly with resolution of hematuria, and the patient was subsequently discharged home after 1 week.

Hematuria recurred 2 weeks post discharge. Repeat cystoscopy showed hemorrhagic mucosa of the bladder neck and prostatic urethra. The areas were cauterized, after which hematuria ceased. The patient was treated with 3 days of infusional cytarabine $100 \mathrm{mg} / \mathrm{m}^{2}$ and continued on daily venetoclax. Hematuria and peripheral blasts transiently resolved, but within 2 weeks, he suffered recurrent hematuria, rising blast count, and worsening cytopenia. He received 3 additional cycles of infusional cytarabine with recurrence of hematuria and rising blast count within days of completing treatment. Goals of care were discussed, and the patient elected to receive hospice care. He died within a week of discharge, $<2$ months after the diagnosis of MS and 2 years after diagnosis of AML.

\section{Discussion}

The genitourinary tract is rarely involved by MS. The kidney, bladder, prostate, testes, epididymis, and uterine cervix are among the documented sites of genitourinary involvement [11-15]. Patients with lower urinary tract MS can present with signs and symptoms related to urinary tract obstruction or urinary retention [16]. It is possible for MS to be misdiagnosed as a primary urothelial malignancy and treated as such [12].

\section{Pathology}

There are no specific gross cystoscopy findings for MS of the urinary bladder, so diagnosis requires histopathological confirmation. The characteristic microscopic growth pattern of myeloid cells is either a diffuse or an Indian file pattern, and the KI-67/MIBI is usually high, ranging from $50 \%$ to $95 \%$. It is subclassified into granulocytic, monoblastic, or myelomonocytic based on the cell type or classified into immature, mature, and blastic types based on the cell maturation. The most common positive markers in paraffin sections include CD68/KP1, MP0, CD117, CD99, CD68/PG-M1, lysozyme, CD34, TdT, CD56, CD61, CD30, glycophorin, and CD4. CD13, CD33, CD117, and MP0 are the most common markers by flow cytometry in tumors with myeloid differentiation and CD14, CD163, and CD11c in tumors with monoblastic differentiation. CD20, CD45RO, CD79a, and CD3 should also be added to the panel in order to exclude other differential diagnoses [4]. Diagnosis of MS in patients with known hematological malignancy may be straightforward, but de novo MS will need bone marrow biopsy and aspiration to assess for other hematological malignancies [17].

\section{Prognosis}

Published clinical outcomes of patients with MS relative to their non-MS AML counterparts are varied. One study reported longer event-free survival and overall survival [18], while another demonstrated comparable outcomes to patients with non-MS AML [19]. In AML patients with $t(8 ; 21)$, shorter overall survival has been observed in those who have an EM component [20]. Given the rarity of this disease, most of the studies are limited by their retrospective design and small size.

\section{Karger'}


Treatment

There is no consensus about the optimal treatment of MS with recommendations based on results of case series and case reports. High-dose chemotherapy, radiation, surgical resection, and allogeneic stem cell transplantation are all modalities that can be incorporated into the therapy of MS. A retrospective study with 24 patients showed significantly longer survival in patients treated with chemotherapy than those who did not (5 years OS $24.3 \%$ vs. $0 \%, p=0.0009$ ) [21]. In a retrospective study of 74 patients with de novo MS, $81 \%$ of patients who did not receive systemic treatment progressed to AML in the first 11 months [22]. The time to progression was longer in those who received a cytarabine-based regimen than other chemotherapy, such as COP (cyclophosphamide, vincristine, and prednisone) or an anthracycline-based regimen. Allogeneic stem cell transplant (alloHSCT) performed early in the course of MS has been shown to prolong survival and is recommended in those who are eligible [23].

Our patient had poor performance status and multiple comorbid conditions that made him ineligible for alloHSCT. He received infusional cytarabine and venetoclax with a brief response, followed by recurrent lower genitourinary symptoms and progressive hematologic disease within weeks.

There are no clear data regarding the optimal consolidation therapies following an initial response. AlloHSCT remains the optimal consolidation therapeutic option, but this may be prohibitive for several AML patients with MS due to their performance status and comorbid conditions. Surgery and radiotherapy do not prolong overall survival but are commonly used to palliate symptoms related to organ dysfunction and obstruction or if there is an incomplete response to chemotherapy [21]. Local control measures, such as cauterization, may be beneficial for symptom control, as was the case in our patient. The role and response rates with newer AML directed therapy are unknown and maybe largely limited to case reports such as this one.

\section{Summary}

The low frequency of MS involving the urinary bladder leads to a lack of consensus treatment guidelines. Our case adds to the sparse evidence available for the management of this condition. Recent advances in allogeneic stem cell transplant and AML-directed therapy will result in patients living longer with AML, which may potentially increase the incidence of EM disease. This calls for increased awareness to ensure prompt diagnosis, enrollment of patients with MS in clinical trials, and focus on MS-specific research to improve the outcomes of patients afflicted by this rare condition. There is a need to evaluate the efficacy of new AMLdirected agents in this disease entity.

\section{Statement of Ethics}

The patient's health-care proxy has given their written informed consent to publish their case (including publication of images). This study is exempt from IRB approval based on our institutional case report policy.

\section{Conflict of Interest Statement}

The authors have no conflicts of interest to declare.

\section{Karger'}




\section{Case Reports in Oncology}

\begin{tabular}{l|l}
\hline Case Rep Oncol 2021;14:1366-1372 \\
\hline DOI: 10.1159/000518529 & $\begin{array}{l}\text { ○ 2021 The Author(s). Published by S. Karger AG, Basel } \\
\text { www.karger.com/cro }\end{array}$ \\
\hline
\end{tabular}

Pearson et al.: Chloroma of the Bladder

\section{Funding Sources}

The authors did not receive any funding.

\section{Author Contributions}

Dr. Laurie K. Pearson and Dr. Ruchi Hamal wrote and edited the manuscript. Dr. Kenneth B. Miller and Dr. Felix Mensah reviewed and edited the manuscript. Dr. Sanhong Yu provided images for the figure, wrote pathology description and discussion, and edited the manuscript.

\section{Data Availability Statement}

All data generated or analyzed during this study are included in this article. Further inquiries can be directed to the corresponding author.

\section{References}

1 Burns A. Observations on the surgical anatomy of the head and neck. Edinburgh, UK: Thomas Royce and Company; 1811.

2 King A. Case of chloroma. Mon J Med Sci. 1853;8(44):97-104.

3 Rappaport H. Tumors of the hematopoietic system. Atlas of tumor pathology. Washington, DC: Armed Forces Institute of Pathology; 1966. p. 241-3.

4 Pileri SA, Ascani S, Cox MC, Campidelli C, Bacci F, Piccioli M, et al. Myeloid sarcoma: clinico-pathologic, phenotypic and cytogenetic analysis of 92 adult patients. Leukemia. 2007 Feb;21(2):340-50.

5 Vardiman JW, Thiele J, Arber DA, Brunning RD, Borowitz MJ, Porwit A, et al. The 2008 revision of the World Health Organization (WHO) classification of myeloid neoplasms and acute leukemia: rationale and important changes. Blood. 2009 Jul 30;114(5):937-51.

6 Neiman RS, Barcos M, Berard C, Bonner H, Mann R, Rydell RE, et al. Granulocytic sarcoma: a clinicopathologic study of 61 biopsied cases. Cancer. 1981 Sep 15;48(6):1426-37.

7 Avni B, Koren-Michowitz M. Myeloid sarcoma: current approach and therapeutic options. Ther Adv Hematol. 2011 Oct;2(5):309-16.

8 Dores GM, Devesa SS, Curtis RE, Linet MS, Morton LM. Acute leukemia incidence and patient survival among children and adults in the United States, 2001-2007. Blood. 2012 Jan 5;119(1):34-43.

9 Byrd JC, Edenfield WJ, Shields DJ, Dawson NA. Extramedullary myeloid cell tumors in acute nonlymphocytic leukemia: a clinical review. J Clin Oncol. 1995 Jul;13(7):1800-16.

10 Cunningham I. Extramedullary sites of leukemia relapse after transplant. Leuk Lymphoma. 2006 Sep;47(9): 1754-67.

11 Frame R, Head D, Lee R, Craven C, Ward JH. Granulocytic sarcoma of the prostate. Two cases causing urinary obstruction. Cancer. 1987 Jan 1;59(1):142-6.

12 Aki H, Baslar Z, Uygun N, Ozguroglu M, Tuzuner N. Primary granulocytic sarcoma of the urinary bladder: case report and review of the literature. Urology. 2002 Aug;60(2):345.

13 Agrawal V, Gupta A, Gupta R, Sharma MC, Das P. Nonleukemic granulocytic sarcoma of kidney with mixed phenotype blasts: a diagnostic dilemma. Indian J Pathol Microbiol. 2011 Jul-Sep;54(3):606-8.

14 Gui W, Li J, Zhang Z, Wang L, Zhao J, Ma L, et al. Primary hematological malignancy of the uterine cervix: a case report. Oncol Lett. 2019 Sep;18(3):3337-41.

15 Sahu KK, Sherif AA, Mishra AK, Lal A, Singh A. Testicular myeloid sarcoma: a systematic review of the literature. Clin Lymphoma Myeloma Leuk. 2019 Oct;19(10):603-18.

16 Steinbock GS, Morrisseau PM, Vinson RK. Acute obstructive renal failure secondary to granulocytic sarcoma (chloroma). Urology. 1986 Mar;27(3):268-70.

17 Bakst RL, Tallman MS, Douer D, Yahalom J. How I treat extramedullary acute myeloid leukemia. Blood. 2011 Oct 6;118(14):3785-93.

18 Tsimberidou AM, Kantarjian HM, Wen S, Keating MJ, O’Brien S, Brandt M, et al. Myeloid sarcoma is associated with superior event-free survival and overall survival compared with acute myeloid leukemia. Cancer. 2008 Sep 15;113(6):1370-8.

19 Avni B, Rund D, Levin M, Grisariu S, Ben-Yehuda D, Bar-Cohen S, et al. Clinical implications of acute myeloid leukemia presenting as myeloid sarcoma. Hematol Oncol. 2012 Mar;30(1):34-40. 
20 Byrd JC, Weiss RB, Arthur DC, Lawrence D, Baer MR, Davey F, et al. Extramedullary leukemia adversely affects hematologic complete remission rate and overall survival in patients with $t(8 ; 21)(q 22 ; q 22)$ : results from Cancer and Leukemia Group B 8461. J Clin Oncol. 1997 Feb;15(2):466-75.

21 Lan TY, Lin DT, Tien HF, Yang RS, Chen CY, Wu K. Prognostic factors of treatment outcomes in patients with granulocytic sarcoma. Acta Haematol. 2009;122(4):238-46.

22 Yamauchi K, Yasuda M. Comparison in treatments of nonleukemic granulocytic sarcoma: report of two cases and a review of 72 cases in the literature. Cancer. 2002 Mar 15;94(6):1739-46.

23 Chevallier P, Mohty M, Lioure B, Michel G, Contentin N, Deconinck E, et al. Allogeneic hematopoietic stem-cell transplantation for myeloid sarcoma: a retrospective study from the SFGM-TC. J Clin Oncol. 2008 Oct 20; 26(30):4940-3. 